Acta Crystallographica Section E

Structure Reports

Online

ISSN 1600-5368

\section{2-(2-Chloropyridin-3-yl)-N-ethyl-4- methyl-1,3-oxazole-5-carboxamide}

\section{Guiqiu Yang, ${ }^{a *}$ Jiakuang Liang, ${ }^{a}$ Haibo $\mathrm{Yu}^{\mathrm{b}}$ and Bin $\mathrm{Li}^{\mathrm{b}}$}

ashenyang Universtity of Chemical Technology, Shenyang 110142, People's Republic of China, and ${ }^{\mathbf{b}}$ Agrochemicals Division, Shenyang Research Institute of Chemical Industry, Shenyang 110021, People's Republic of China

Correspondence e-mail: yangguiqiu@gmail.com

Received 20 October 2010; accepted 8 November 2010

Key indicators: single-crystal X-ray study; $T=296 \mathrm{~K}$; mean $\sigma(\mathrm{C}-\mathrm{C})=0.003 \AA$; $R$ factor $=0.039 ; w R$ factor $=0.109 ;$ data-to-parameter ratio $=13.2$.

In the title compound, $\mathrm{C}_{12} \mathrm{H}_{12} \mathrm{ClN}_{3} \mathrm{O}_{2}$, the dihedral angle between the aromatic rings is $8.42(10)^{\circ}$. In the crystal, molecules are linked by $\mathrm{N}-\mathrm{H} \cdots \mathrm{O}$ hydrogen bonds, generating $C(4)$ chains propagating in [001].

\section{Related literature}

For background to derivatives of oxazolyl carboxylic acids, see: Takechi et al. (2000); Lechel et al. (2009).

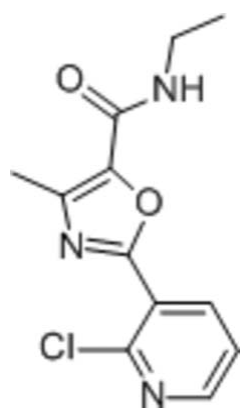

\section{Experimental}

Crystal data

$\mathrm{C}_{12} \mathrm{H}_{12} \mathrm{ClN}_{3} \mathrm{O}_{2} \quad M_{r}=265.70$
Monoclinic, $P 2_{1} / c$

$a=8.2143(12) \AA$

$b=14.545$ (2) $\AA$

$c=10.4360(16) \AA$

$\beta=97.425$ (3)

$V=1236.4(3) \AA^{3}$

Data collection

Bruker SMART CCD

diffractometer

Absorption correction: multi-scan

(SADABS; Bruker, 2001)

$T_{\text {min }}=0.908, T_{\max }=0.936$

Refinement

$R\left[F^{2}>2 \sigma\left(F^{2}\right)\right]=0.039$

$w R\left(F^{2}\right)=0.109$

$S=1.07$

2183 reflections

$Z=4$

Mo $K \alpha$ radiation

$\mu=0.31 \mathrm{~mm}^{-1}$

$T=296 \mathrm{~K}$

$0.32 \times 0.28 \times 0.22 \mathrm{~mm}$

6234 measured reflections 2183 independent reflections 1736 reflections with $I>2 \sigma(I)$

$R_{\text {int }}=0.027$

Table 1

Hydrogen-bond geometry $\left(\AA{ }^{\circ}\right)$.

\begin{tabular}{lllll}
\hline$D-\mathrm{H} \cdots A$ & $D-\mathrm{H}$ & $\mathrm{H} \cdots A$ & $D \cdots A$ & $D-\mathrm{H} \cdots A$ \\
\hline $\mathrm{N} 3-\mathrm{H} 3 \cdots \mathrm{O} 2{ }^{\mathrm{i}}$ & 0.86 & 2.29 & $3.115(2)$ & 161 \\
\hline
\end{tabular}

Symmetry code: (i) $x,-y+\frac{3}{2}, z+\frac{1}{2}$.

Data collection: SMART (Bruker, 2001); cell refinement: SAINT (Bruker, 2001); data reduction: $S A I N T$; program(s) used to solve structure: SHELXS97 (Sheldrick, 2008); program(s) used to refine structure: SHELXL97 (Sheldrick, 2008); molecular graphics: SHELXTL (Sheldrick, 2008); software used to prepare material for publication: SHELXL97.

Supplementary data and figures for this paper are available from the IUCr electronic archives (Reference: HB5697).

\section{References}

Bruker (2001). SAINT and SMART. Bruker AXS Inc., Madison, Wisconsin, USA.

Lechel, T., Lentz, D. \& Reissig, H. U. (2009). Chem. Eur. J. 15, 5432-5435.

Sheldrick, G. M. (2008). Acta Cryst. A64, 112-122.

Takechi, H., Oda, Y., Nishizono, N., Oda, K. \& Machida, M. (2000). Chem. Pharm. Bull. 48, 1702-1710. 


\section{supporting information}

Acta Cryst. (2010). E66, o3154 [https://doi.org/10.1107/S1600536810045885]

\section{2-(2-Chloropyridin-3-yl)-N-ethyl-4-methyl-1,3-oxazole-5-carboxamide}

\section{Guiqiu Yang, Jiakuang Liang, Haibo Yu and Bin Li}

\section{S1. Comment}

Derivatives of oxazolyl carboxylic acid are important heterocyclic compounds. They display a broad range of biological, medical and pharmacological properties (Takechi et al., 2000; Lechel et al., 2009). We report the crystal structure of the title compound (I) to determine the structure of the main product in the preparation of derivatives ofoxazolyl carboxylic acid. The molecular structure of (I) (Fig. 1) contains no crystallographically imposed symmetry. The pyridine and oxazole rings in each of the ligands are not coplanar, the dihedral angle formed by the least-squares planes of the benzene and pyrazole rings being equal to $8.8^{\circ}$. Analysis of the crystal packing of (I) shows the existence of $\mathrm{N} 3-\mathrm{H} 3 \cdots \mathrm{O} 2$ interactions, as shown in Fig. 2.

\section{S2. Experimental}

The title compound was synthesized by 2-(2-chloropyridin-3-yl) -4-methyloxazole-5-carbonyl chloride with ethanamine in toluene. The crude products were purified by silica-gel column chromatography and then grown from dichloromethane to afford colorless blocks of (I). To a $100 \mathrm{ml}$ flask ethanamine $(0.24 \mathrm{~g}, 5.40 \mathrm{mmol})$,triethylamine $(0.68 \mathrm{~g}, 6.75 \mathrm{mmol})$, 2-(2-chloropyridin-3-yl)-4-methyloxazole-5-carbonyl chloride (1.16 g, $4.50 \mathrm{mmol})$ and $45 \mathrm{ml}$ toluene were added sequentially. The reaction mixture was reacted for $2 \mathrm{~h}$. After separation through silica gel column chromatography (fluent: ethyl acetate/petroleum ether=1/5), The title compound was gained as a yellow solid $(0.42 \mathrm{~g}, 58 \%)$.

Anal. Calcd for $\mathrm{C}_{12} \mathrm{H}_{12} \mathrm{~N}_{3}: \mathrm{C}, 54.25 ; \mathrm{H}, 4.55 ; \mathrm{N}, 15.82$. Found: $\mathrm{C}, 54.33 ; \mathrm{H}, 4.54 ; \mathrm{N}, 15.75 .{ }^{1} \mathrm{H} \mathrm{NMR}\left(\mathrm{CDCl}_{3}\right): 1.27$ $\left(\mathrm{t}, 3 \mathrm{H}, \mathrm{CH}_{3}\right), 2.61\left(\mathrm{~s}, 3 \mathrm{H}, \mathrm{Ar}-\mathrm{CH}_{3}\right), 3.50\left(\mathrm{~m}, 2 \mathrm{H}, \mathrm{CH}_{2}\right), 6.29(\mathrm{br} \mathrm{s}, 1 \mathrm{H}, \mathrm{NH}), 7.40(\mathrm{dd}, 1 \mathrm{H}, \mathrm{py}-\mathrm{H}), 8.42(\mathrm{dd}, 1 \mathrm{H}, \mathrm{py}-\mathrm{H})$, $8.53(\mathrm{dd}, 1 \mathrm{H}, \mathrm{py}-\mathrm{H})$.

\section{S3. Refinement}

Although all $\mathrm{H}$ atoms were visible in difference maps, they were finally placed in geometrically calculated positions, with C-Hdistances in the range $0.93-0.97 \AA$ and $\mathrm{N}-\mathrm{H}$ distances of $0.86 \AA$, andincluded in the final refinement in the riding model approximation, with $U_{\text {iso }}(\mathrm{H})=1.2 U_{\text {eq }}(\mathrm{C}, \mathrm{N})$ and $U_{\text {iso }}(\mathrm{H})=1.5 U_{\text {eq }}(\mathrm{C})$. 


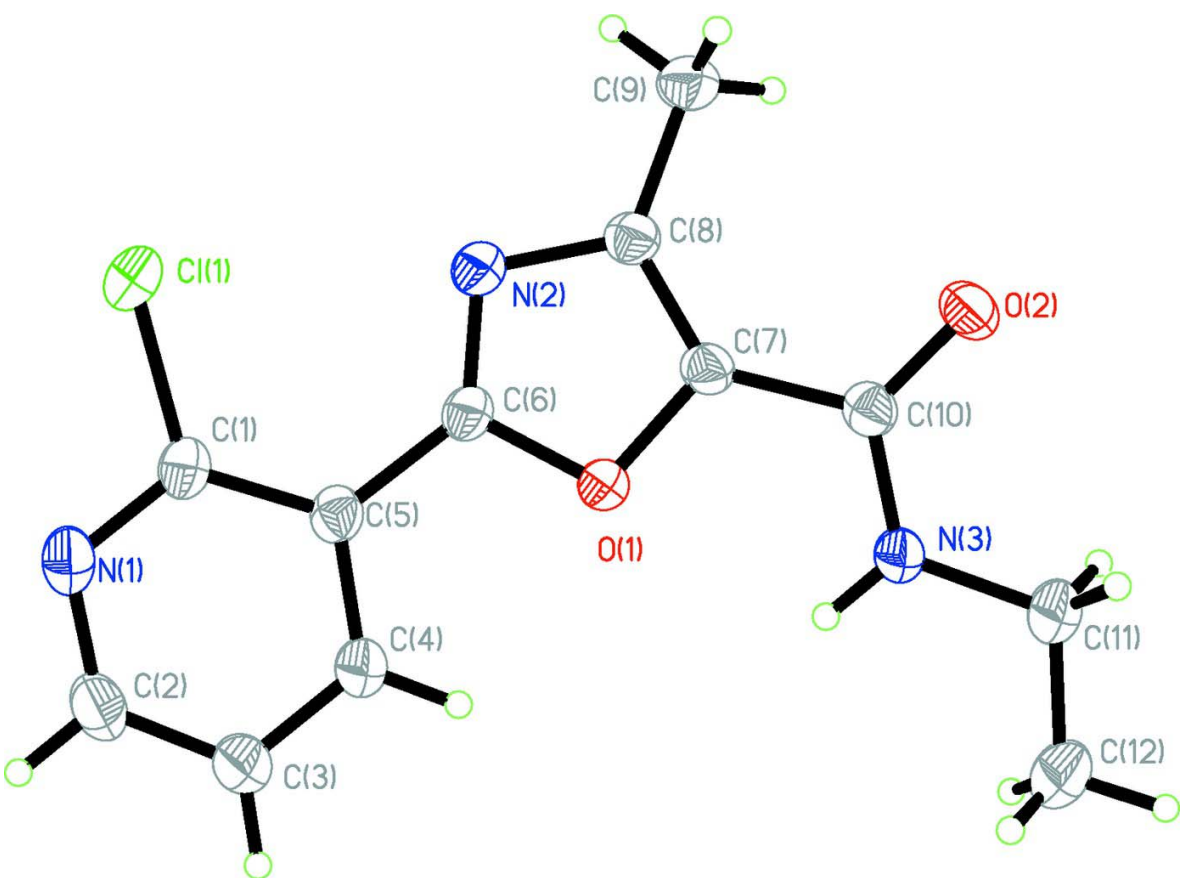

Figure 1

The molecular structure of (I), with atom labels and 30\% probability displacement ellipsoids.

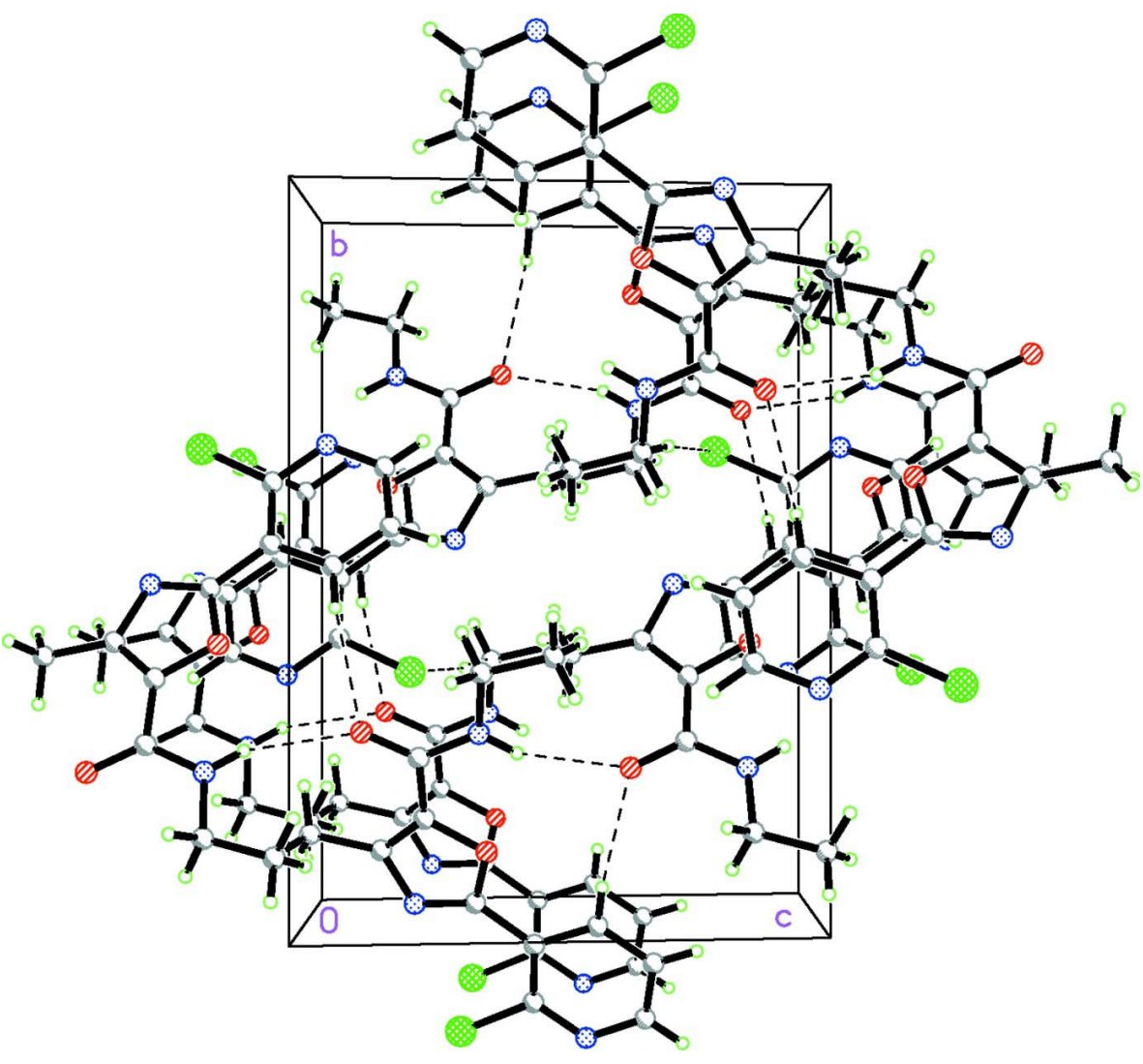


Figure 2

Crystal packing of (I).

2-(2-Chloropyridin-3-yl)-N-ethyl-4-methyl-1,3-oxazole-5-carboxamide

Crystal data

$\mathrm{C}_{12} \mathrm{H}_{12} \mathrm{ClN}_{3} \mathrm{O}_{2}$

$M_{r}=265.70$

Monoclinic, $P 2_{1} / c$

$a=8.2143$ (12) $\AA$

$b=14.545(2) \AA$

$c=10.4360(16) \AA$

$\beta=97.425(3)^{\circ}$

$V=1236.4(3) \AA^{3}$

$Z=4$

$F(000)=552$

$D_{\mathrm{x}}=1.427 \mathrm{Mg} \mathrm{m}^{-3}$

Mo $K \alpha$ radiation, $\lambda=0.71073 \AA$

Cell parameters from 2185 reflections

$\theta=2.4-25.4^{\circ}$

$\mu=0.31 \mathrm{~mm}^{-1}$

$T=296 \mathrm{~K}$

Block, colorless

$0.32 \times 0.28 \times 0.22 \mathrm{~mm}$

\section{Data collection}

\section{Bruker SMART CCD}

diffractometer

Radiation source: fine-focus sealed tube

Graphite monochromator

$\omega$ scans

Absorption correction: multi-scan

(SADABS; Bruker, 2001)

$T_{\min }=0.908, T_{\max }=0.936$

\section{Refinement}

Refinement on $F^{2}$

Least-squares matrix: full

$R\left[F^{2}>2 \sigma\left(F^{2}\right)\right]=0.039$

$w R\left(F^{2}\right)=0.109$

$S=1.07$

2183 reflections

165 parameters

0 restraints

Primary atom site location: structure-invariant direct methods
6234 measured reflections

2183 independent reflections

1736 reflections with $I>2 \sigma(I)$

$R_{\text {int }}=0.027$

$\theta_{\max }=25.0^{\circ}, \theta_{\min }=2.4^{\circ}$

$h=-9 \rightarrow 9$

$k=-17 \rightarrow 17$

$l=-12 \rightarrow 8$

Secondary atom site location: difference Fourier map

Hydrogen site location: inferred from neighbouring sites

$\mathrm{H}$-atom parameters constrained

$w=1 /\left[\sigma^{2}\left(F_{\mathrm{o}}^{2}\right)+(0.0583 P)^{2}+0.2339 P\right]$

where $P=\left(F_{\mathrm{o}}{ }^{2}+2 F_{\mathrm{c}}{ }^{2}\right) / 3$

$(\Delta / \sigma)_{\max }<0.001$

$\Delta \rho_{\max }=0.23 \mathrm{e} \AA^{-3}$

$\Delta \rho_{\min }=-0.23 \mathrm{e} \AA^{-3}$

Special details

Geometry. All esds (except the esd in the dihedral angle between two 1.s. planes) are estimated using the full covariance matrix. The cell esds are taken into account individually in the estimation of esds in distances, angles and torsion angles; correlations between esds in cell parameters are only used when they are defined by crystal symmetry. An approximate (isotropic) treatment of cell esds is used for estimating esds involving l.s. planes.

Refinement. Refinement of $\mathrm{F}^{2}$ against ALL reflections. The weighted R-factor $\mathrm{wR}$ and goodness of fit $\mathrm{S}$ are based on $\mathrm{F}^{2}$, conventional R-factors $R$ are based on $F$, with $F$ set to zero for negative $F^{2}$. The threshold expression of $F^{2}>2 \operatorname{sigma}\left(\mathrm{F}^{2}\right)$ is used only for calculating R-factors(gt) etc. and is not relevant to the choice of reflections for refinement. R-factors based on $\mathrm{F}^{2}$ are statistically about twice as large as those based on F, and R- factors based on ALL data will be even larger.

Fractional atomic coordinates and isotropic or equivalent isotropic displacement parameters $\left(\hat{A}^{2}\right)$

\begin{tabular}{lllll}
\hline & $x$ & $y$ & $z$ & $U_{\text {iso }} * / U_{\text {eq }}$ \\
\hline C11 & $0.52313(8)$ & $1.15459(4)$ & $0.30039(6)$ & $0.0650(2)$ \\
O1 & $0.81459(15)$ & $0.89027(8)$ & $0.35667(12)$ & $0.0424(3)$
\end{tabular}




$\begin{array}{lllll}\mathrm{O} 2 & 0.91578(18) & 0.72507(9) & 0.12724(13) & 0.0542(4) \\ \mathrm{N} 1 & 0.6093(2) & 1.15962(11) & 0.54565(19) & 0.0582(5) \\ \mathrm{N} 2 & 0.6424(2) & 0.96872(10) & 0.21507(15) & 0.0449(4) \\ \mathrm{N} 3 & 0.9699(2) & 0.72633(10) & 0.34513(15) & 0.0476(4) \\ \mathrm{H} 3 & 0.9523 & 0.7530 & 0.4156 & 0.057^{*} \\ \mathrm{C} 1 & 0.6202(2) & 1.10823(12) & 0.4431(2) & 0.0457(5) \\ \mathrm{C} 2 & 0.6806(3) & 1.12864(15) & 0.6593(2) & 0.0625(6) \\ \mathrm{H} 2 & 0.6733 & 1.1642 & 0.7324 & 0.075^{*} \\ \mathrm{C} 3 & 0.7641(3) & 1.04702(15) & 0.6739(2) & 0.0636(6) \\ \mathrm{H} 3 \mathrm{~A} & 0.8122 & 1.0278 & 0.7551 & 0.076^{*} \\ \mathrm{C} 4 & 0.7752(3) & 0.99428(13) & 0.56647(19) & 0.0525(5) \\ \mathrm{H} 4 & 0.8320 & 0.9388 & 0.5744 & 0.063^{*} \\ \mathrm{C} 5 & 0.7019(2) & 1.02335(12) & 0.44587(18) & 0.0420(4) \\ \mathrm{C} 6 & 0.7133(2) & 0.96435(12) & 0.33294(18) & 0.0399(4) \\ \mathrm{C} 7 & 0.8032(2) & 0.84506(11) & 0.23930(18) & 0.0402(4) \\ \mathrm{C} 8 & 0.6982(2) & 0.89204(12) & 0.15339(18) & 0.0416(4) \\ \mathrm{C} 9 & 0.6388(3) & 0.87207(15) & 0.0153(2) & 0.0567(6) \\ \mathrm{H} 9 \mathrm{~A} & 0.7179 & 0.8936 & -0.0378 & 0.085^{*} \\ \mathrm{H} 9 \mathrm{~B} & 0.5359 & 0.9027 & -0.0091 & 0.085^{*} \\ \mathrm{H} 9 \mathrm{C} & 0.6242 & 0.8070 & 0.0038 & 0.085^{*} \\ \mathrm{C} 10 & 0.9008(2) & 0.76014(12) & 0.23233(18) & 0.0412(4) \\ \mathrm{C} 11 & 1.0747(3) & 0.64501(15) & 0.3517(2) & 0.0627(6) \\ \mathrm{H} 11 \mathrm{~A} & 1.0109 & 0.5931 & 0.3149 & 0.075^{*} \\ \mathrm{H} 11 \mathrm{~B} & 1.1631 & 0.6554 & 0.3001 & 0.075^{*} \\ \mathrm{C} 12 & 1.1446(4) & 0.6227(2) & 0.4837(3) & 0.0857(9) \\ \mathrm{H} 12 \mathrm{~A} & 1.2037 & 0.6749 & 0.5220 & 0.129^{*} \\ \mathrm{H} 12 \mathrm{~B} & 1.2181 & 0.5715 & 0.4827 & 0.129^{*} \\ \mathrm{H} 12 \mathrm{C} & 1.0580 & 0.6070 & 0.5333 & 0.129^{*}\end{array}$

Atomic displacement parameters $\left(\AA^{2}\right)$

\begin{tabular}{lllllll}
\hline & $U^{11}$ & $U^{22}$ & $U^{33}$ & $U^{12}$ & $U^{13}$ & $U^{23}$ \\
\hline C11 & $0.0845(4)$ & $0.0471(3)$ & $0.0613(4)$ & $0.0194(3)$ & $0.0012(3)$ & $0.0078(2)$ \\
O1 & $0.0535(8)$ & $0.0366(7)$ & $0.0360(7)$ & $0.0063(6)$ & $0.0015(6)$ & $-0.0004(5)$ \\
O2 & $0.0808(10)$ & $0.0446(8)$ & $0.0379(8)$ & $0.0047(7)$ & $0.0104(7)$ & $-0.0043(6)$ \\
N1 & $0.0718(12)$ & $0.0405(9)$ & $0.0626(12)$ & $0.0066(8)$ & $0.0098(10)$ & $-0.0062(8)$ \\
N2 & $0.0531(10)$ & $0.0376(8)$ & $0.0428(9)$ & $0.0036(7)$ & $0.0016(8)$ & $0.0025(7)$ \\
N3 & $0.0633(10)$ & $0.0408(9)$ & $0.0387(9)$ & $0.0139(7)$ & $0.0071(8)$ & $0.0002(7)$ \\
C1 & $0.0502(11)$ & $0.0347(10)$ & $0.0524(12)$ & $0.0005(8)$ & $0.0078(9)$ & $0.0015(8)$ \\
C2 & $0.0843(16)$ & $0.0496(12)$ & $0.0541(14)$ & $0.0016(12)$ & $0.0106(12)$ & $-0.0126(11)$ \\
C3 & $0.0910(17)$ & $0.0517(13)$ & $0.0461(13)$ & $0.0102(12)$ & $0.0011(12)$ & $-0.0032(10)$ \\
C4 & $0.0688(14)$ & $0.0396(10)$ & $0.0481(12)$ & $0.0090(9)$ & $0.0034(10)$ & $-0.0018(9)$ \\
C5 & $0.0474(11)$ & $0.0339(9)$ & $0.0453(11)$ & $-0.0019(8)$ & $0.0080(9)$ & $0.0023(8)$ \\
C6 & $0.0454(10)$ & $0.0308(9)$ & $0.0431(11)$ & $0.0008(7)$ & $0.0048(9)$ & $0.0039(7)$ \\
C7 & $0.0502(11)$ & $0.0345(9)$ & $0.0353(10)$ & $-0.0022(8)$ & $0.0029(8)$ & $-0.0011(7)$ \\
C8 & $0.0479(11)$ & $0.0375(10)$ & $0.0387(10)$ & $-0.0033(8)$ & $0.0031(8)$ & $0.0016(8)$ \\
C9 & $0.0672(14)$ & $0.0549(12)$ & $0.0442(12)$ & $0.0043(11)$ & $-0.0069(10)$ & $-0.0020(10)$ \\
C10 & $0.0500(11)$ & $0.0348(9)$ & $0.0392(10)$ & $-0.0035(8)$ & $0.0072(9)$ & $-0.0006(8)$
\end{tabular}




\begin{tabular}{lllllll}
$\mathrm{C} 11$ & $0.0796(16)$ & $0.0500(13)$ & $0.0578(14)$ & $0.0237(11)$ & $0.0068(12)$ & $0.0004(10)$ \\
$\mathrm{C} 12$ & $0.103(2)$ & $0.0863(18)$ & $0.0661(17)$ & $0.0496(16)$ & $0.0029(15)$ & $0.0079(14)$ \\
\hline
\end{tabular}

Geometric parameters $\left(A,{ }^{\circ}\right)$

\begin{tabular}{|c|c|c|c|}
\hline $\mathrm{C} 11-\mathrm{C} 1$ & $1.733(2)$ & $\mathrm{C} 4-\mathrm{C} 5$ & $1.389(3)$ \\
\hline $\mathrm{O} 1-\mathrm{C} 6$ & $1.364(2)$ & $\mathrm{C} 4-\mathrm{H} 4$ & 0.9300 \\
\hline $\mathrm{O} 1-\mathrm{C} 7$ & $1.383(2)$ & $\mathrm{C} 5-\mathrm{C} 6$ & $1.471(3)$ \\
\hline $\mathrm{O} 2-\mathrm{C} 10$ & $1.230(2)$ & $\mathrm{C} 7-\mathrm{C} 8$ & $1.347(2)$ \\
\hline $\mathrm{N} 1-\mathrm{C} 1$ & $1.318(3)$ & $\mathrm{C} 7-\mathrm{C} 10$ & $1.479(3)$ \\
\hline $\mathrm{N} 1-\mathrm{C} 2$ & $1.332(3)$ & $\mathrm{C} 8-\mathrm{C} 9$ & $1.489(3)$ \\
\hline $\mathrm{N} 2-\mathrm{C} 6$ & $1.293(2)$ & C9-H9A & 0.9600 \\
\hline $\mathrm{N} 2-\mathrm{C} 8$ & $1.395(2)$ & $\mathrm{C} 9-\mathrm{H} 9 \mathrm{~B}$ & 0.9600 \\
\hline $\mathrm{N} 3-\mathrm{C} 10$ & $1.333(2)$ & $\mathrm{C} 9-\mathrm{H} 9 \mathrm{C}$ & 0.9600 \\
\hline $\mathrm{N} 3-\mathrm{C} 11$ & $1.459(2)$ & $\mathrm{C} 11-\mathrm{C} 12$ & $1.459(3)$ \\
\hline $\mathrm{N} 3-\mathrm{H} 3$ & 0.8600 & $\mathrm{C} 11-\mathrm{H} 11 \mathrm{~A}$ & 0.9700 \\
\hline $\mathrm{C} 1-\mathrm{C} 5$ & $1.404(3)$ & $\mathrm{C} 11-\mathrm{H} 11 \mathrm{~B}$ & 0.9700 \\
\hline $\mathrm{C} 2-\mathrm{C} 3$ & $1.370(3)$ & $\mathrm{C} 12-\mathrm{H} 12 \mathrm{~A}$ & 0.9600 \\
\hline $\mathrm{C} 2-\mathrm{H} 2$ & 0.9300 & $\mathrm{C} 12-\mathrm{H} 12 \mathrm{~B}$ & 0.9600 \\
\hline $\mathrm{C} 3-\mathrm{C} 4$ & $1.372(3)$ & $\mathrm{C} 12-\mathrm{H} 12 \mathrm{C}$ & 0.9600 \\
\hline $\mathrm{C} 3-\mathrm{H} 3 \mathrm{~A}$ & 0.9300 & & \\
\hline $\mathrm{C} 6-\mathrm{O} 1-\mathrm{C} 7$ & $104.17(14)$ & $\mathrm{O} 1-\mathrm{C} 7-\mathrm{C} 10$ & $117.74(15)$ \\
\hline $\mathrm{C} 1-\mathrm{N} 1-\mathrm{C} 2$ & $117.59(18)$ & $\mathrm{C} 7-\mathrm{C} 8-\mathrm{N} 2$ & $108.60(16)$ \\
\hline $\mathrm{C} 6-\mathrm{N} 2-\mathrm{C} 8$ & $105.37(15)$ & $\mathrm{C} 7-\mathrm{C} 8-\mathrm{C} 9$ & $130.30(18)$ \\
\hline $\mathrm{C} 10-\mathrm{N} 3-\mathrm{C} 11$ & $121.45(17)$ & $\mathrm{N} 2-\mathrm{C} 8-\mathrm{C} 9$ & 121.08 \\
\hline $\mathrm{C} 10-\mathrm{N} 3-\mathrm{H} 3$ & 119.3 & $\mathrm{C} 8-\mathrm{C} 9-\mathrm{H} 9 \mathrm{~A}$ & 109.5 \\
\hline $\mathrm{C} 11-\mathrm{N} 3-\mathrm{H} 3$ & 119.3 & $\mathrm{C} 8-\mathrm{C} 9-\mathrm{H} 9 \mathrm{~B}$ & 109.5 \\
\hline $\mathrm{N} 1-\mathrm{C} 1-\mathrm{C} 5$ & $124.39(19)$ & $\mathrm{H} 9 \mathrm{~A}-\mathrm{C} 9-\mathrm{H} 9 \mathrm{~B}$ & 109.5 \\
\hline $\mathrm{N} 1-\mathrm{C} 1-\mathrm{Cl} 1$ & $113.91(15)$ & $\mathrm{C} 8-\mathrm{C} 9-\mathrm{H} 9 \mathrm{C}$ & 109.5 \\
\hline $\mathrm{C} 5-\mathrm{C} 1-\mathrm{Cl} 1$ & $121.70(15)$ & $\mathrm{H} 9 \mathrm{~A}-\mathrm{C} 9-\mathrm{H} 9 \mathrm{C}$ & 109.5 \\
\hline $\mathrm{N} 1-\mathrm{C} 2-\mathrm{C} 3$ & $123.3(2)$ & $\mathrm{H} 9 \mathrm{~B}-\mathrm{C} 9-\mathrm{H} 9 \mathrm{C}$ & 109.5 \\
\hline $\mathrm{N} 1-\mathrm{C} 2-\mathrm{H} 2$ & 118.4 & $\mathrm{O} 2-\mathrm{C} 10-\mathrm{N} 3$ & $123.63(17)$ \\
\hline $\mathrm{C} 3-\mathrm{C} 2-\mathrm{H} 2$ & 118.4 & $\mathrm{O} 2-\mathrm{C} 10-\mathrm{C} 7$ & $120.50(17)$ \\
\hline $\mathrm{C} 2-\mathrm{C} 3-\mathrm{C} 4$ & $118.6(2)$ & $\mathrm{N} 3-\mathrm{C} 10-\mathrm{C} 7$ & $115.86(16)$ \\
\hline $\mathrm{C} 2-\mathrm{C} 3-\mathrm{H} 3 \mathrm{~A}$ & 120.7 & $\mathrm{C} 12-\mathrm{C} 11-\mathrm{N} 3$ & $112.48(19)$ \\
\hline $\mathrm{C} 4-\mathrm{C} 3-\mathrm{H} 3 \mathrm{~A}$ & 120.7 & $\mathrm{C} 12-\mathrm{C} 11-\mathrm{H} 11 \mathrm{~A}$ & 109.1 \\
\hline $\mathrm{C} 3-\mathrm{C} 4-\mathrm{C} 5$ & $120.25(19)$ & $\mathrm{N} 3-\mathrm{C} 11-\mathrm{H} 11 \mathrm{~A}$ & 109.1 \\
\hline $\mathrm{C} 3-\mathrm{C} 4-\mathrm{H} 4$ & 119.9 & $\mathrm{C} 12-\mathrm{C} 11-\mathrm{H} 11 \mathrm{~B}$ & 109.1 \\
\hline $\mathrm{C} 5-\mathrm{C} 4-\mathrm{H} 4$ & 119.9 & N3-C11-H11B & 109.1 \\
\hline $\mathrm{C} 4-\mathrm{C} 5-\mathrm{C} 1$ & $115.87(18)$ & $\mathrm{H} 11 \mathrm{~A}-\mathrm{C} 11-\mathrm{H} 11 \mathrm{~B}$ & 107.8 \\
\hline $\mathrm{C} 4-\mathrm{C} 5-\mathrm{C} 6$ & $118.92(16)$ & $\mathrm{C} 11-\mathrm{C} 12-\mathrm{H} 12 \mathrm{~A}$ & 109.5 \\
\hline $\mathrm{C} 1-\mathrm{C} 5-\mathrm{C} 6$ & $125.21(17)$ & $\mathrm{C} 11-\mathrm{C} 12-\mathrm{H} 12 \mathrm{~B}$ & 109.5 \\
\hline $\mathrm{N} 2-\mathrm{C} 6-\mathrm{O} 1$ & $113.63(16)$ & $\mathrm{H} 12 \mathrm{~A}-\mathrm{C} 12-\mathrm{H} 12 \mathrm{~B}$ & 109.5 \\
\hline $\mathrm{N} 2-\mathrm{C} 6-\mathrm{C} 5$ & $131.78(17)$ & $\mathrm{C} 11-\mathrm{C} 12-\mathrm{H} 12 \mathrm{C}$ & 109.5 \\
\hline $\mathrm{O} 1-\mathrm{C} 6-\mathrm{C} 5$ & $114.57(16)$ & $\mathrm{H} 12 \mathrm{~A}-\mathrm{C} 12-\mathrm{H} 12 \mathrm{C}$ & 109.5 \\
\hline $\mathrm{C} 8-\mathrm{C} 7-\mathrm{O} 1$ & $108.22(15)$ & $\mathrm{H} 12 \mathrm{~B}-\mathrm{C} 12-\mathrm{H} 12 \mathrm{C}$ & 109.5 \\
\hline $\mathrm{C} 8-\mathrm{C} 7-\mathrm{C} 10$ & $134.03(17)$ & & \\
\hline
\end{tabular}




\begin{tabular}{llll}
$\mathrm{C} 2-\mathrm{N} 1-\mathrm{C} 1-\mathrm{C} 5$ & $-0.1(3)$ & $\mathrm{C} 4-\mathrm{C} 5-\mathrm{C} 6-\mathrm{O} 1$ & $-7.8(3)$ \\
$\mathrm{C} 2-\mathrm{N} 1-\mathrm{C} 1-\mathrm{C} 1$ & $179.87(17)$ & $\mathrm{C} 1-\mathrm{C} 5-\mathrm{C} 6-\mathrm{O} 1$ & $172.89(16)$ \\
$\mathrm{C} 1-\mathrm{N} 1-\mathrm{C} 2-\mathrm{C} 3$ & $0.3(4)$ & $\mathrm{C} 6-\mathrm{O} 1-\mathrm{C} 7-\mathrm{C} 8$ & $-0.41(19)$ \\
$\mathrm{N} 1-\mathrm{C} 2-\mathrm{C} 3-\mathrm{C} 4$ & $0.0(4)$ & $\mathrm{C} 6-\mathrm{O} 1-\mathrm{C} 7-\mathrm{C} 10$ & $179.86(15)$ \\
$\mathrm{C} 2-\mathrm{C} 3-\mathrm{C} 4-\mathrm{C} 5$ & $-0.5(4)$ & $\mathrm{O} 1-\mathrm{C} 7-\mathrm{C} 8-\mathrm{N} 2$ & $0.6(2)$ \\
$\mathrm{C} 3-\mathrm{C} 4-\mathrm{C} 5-\mathrm{C} 1$ & $0.6(3)$ & $\mathrm{C} 10-\mathrm{C} 7-\mathrm{C} 8-\mathrm{N} 2$ & $-179.76(19)$ \\
$\mathrm{C} 3-\mathrm{C} 4-\mathrm{C} 5-\mathrm{C} 6$ & $-178.8(2)$ & $\mathrm{O} 1-\mathrm{C} 7-\mathrm{C} 8-\mathrm{C} 9$ & $-178.03(19)$ \\
$\mathrm{N} 1-\mathrm{C} 1-\mathrm{C} 5-\mathrm{C} 4$ & $-0.3(3)$ & $\mathrm{C} 10-\mathrm{C} 7-\mathrm{C} 8-\mathrm{C} 9$ & $1.6(4)$ \\
$\mathrm{C} 11-\mathrm{C} 1-\mathrm{C} 5-\mathrm{C} 4$ & $179.72(15)$ & $\mathrm{C} 6-\mathrm{N} 2-\mathrm{C} 8-\mathrm{C} 7$ & $-0.5(2)$ \\
$\mathrm{N} 1-\mathrm{C} 1-\mathrm{C} 5-\mathrm{C} 6$ & $179.00(19)$ & $\mathrm{C} 6-\mathrm{N} 2-\mathrm{C} 8-\mathrm{C} 9$ & $178.25(18)$ \\
$\mathrm{C} 11-\mathrm{C} 1-\mathrm{C} 5-\mathrm{C} 6$ & $-1.0(3)$ & $\mathrm{C} 11-\mathrm{N} 3-\mathrm{C} 10-\mathrm{O} 2$ & $1.9(3)$ \\
$\mathrm{C} 8-\mathrm{N} 2-\mathrm{C} 6-\mathrm{O} 1$ & $0.2(2)$ & $\mathrm{C} 11-\mathrm{N} 3-\mathrm{C} 10-\mathrm{C} 7$ & $-177.52(18)$ \\
$\mathrm{C} 8-\mathrm{N} 2-\mathrm{C} 6-\mathrm{C} 5$ & $-178.29(19)$ & $\mathrm{C} 8-\mathrm{C} 7-\mathrm{C} 10-\mathrm{O} 2$ & $11.7(3)$ \\
$\mathrm{C} 7-\mathrm{O} 1-\mathrm{C} 6-\mathrm{N} 2$ & $0.1(2)$ & $\mathrm{O} 1-\mathrm{C} 7-\mathrm{C} 10-\mathrm{O} 2$ & $-168.64(17)$ \\
$\mathrm{C} 7-\mathrm{O} 1-\mathrm{C} 6-\mathrm{C} 5$ & $178.89(15)$ & $\mathrm{C} 8-\mathrm{C} 7-\mathrm{C} 10-\mathrm{N} 3$ & $-168.8(2)$ \\
$\mathrm{C} 4-\mathrm{C} 5-\mathrm{C} 6-\mathrm{N} 2$ & $170.7(2)$ & $\mathrm{O} 1-\mathrm{C} 7-\mathrm{C} 10-\mathrm{N} 3$ & $10.8(2)$ \\
$\mathrm{C} 1-\mathrm{C} 5-\mathrm{C} 6-\mathrm{N} 2$ & $-8.6(3)$ & $\mathrm{C} 10-\mathrm{N} 3-\mathrm{C} 11-\mathrm{C} 12$ & $176.9(2)$ \\
\hline
\end{tabular}

Hydrogen-bond geometry $\left(\AA,{ }^{\circ}\right)$

\begin{tabular}{lllll}
\hline$D-\mathrm{H} \cdots A$ & $D-\mathrm{H}$ & $\mathrm{H} \cdots A$ & $D \cdots A$ & $D-\mathrm{H} \cdots A$ \\
\hline $\mathrm{N} 3-\mathrm{H} 3 \cdots \mathrm{O}^{\mathrm{i}}$ & 0.86 & 2.29 & $3.115(2)$ & 161 \\
\hline
\end{tabular}

Symmetry code: (i) $x,-y+3 / 2, z+1 / 2$. 